\title{
ORIGINAL
}

\section{Acute respiratory distress syndrome-attributable mortality in critically ill patients with sepsis}

Catherine L. Auriemma ${ }^{1}{ }^{2 *}$, Hanjing Zhuo ${ }^{3}$, Kevin Delucchi ${ }^{4}$, Thomas Deiss ${ }^{5}$, Tom Liu ${ }^{6}$, Alejandra Jauregui ${ }^{7}$, Serena Ke ${ }^{8}$, Kathryn Vessel ${ }^{7}$, Matthew Lippi ${ }^{9}$, Eric Seeley ${ }^{10}$, Kirsten N. Kangelaris ${ }^{7}$, Antonio Gomez ${ }^{8}$, Carolyn Hendrickson ${ }^{8}$, Kathleen D. Liu ${ }^{11,12}$, Michael A. Matthay ${ }^{3,10,12}$, Lorraine B. Ware ${ }^{13}$ and Carolyn S. Calfee $3,10,12$

(c) 2020 Springer-Verlag GmbH Germany, part of Springer Nature

\begin{abstract}
Purpose: Previous studies assessing impact of acute respiratory distress syndrome (ARDS) on mortality have shown conflicting results. We sought to assess the independent association of ARDS with in-hospital mortality among intensive care unit (ICU) patients with sepsis.

Methods: We studied two prospective sepsis cohorts drawn from the Early Assessment of Renal and Lung Injury (EARLI; $n=474$ ) and Validating Acute Lung Injury markers for Diagnosis (VALID; $n=337$ ) cohorts. ARDS was defined by Berlin criteria. We used logistic regression to compare in-hospital mortality in patients with and without ARDS, controlling for baseline severity of illness. We also estimated attributable mortality, adjusted for illness severity by stratification.
\end{abstract}

Results: ARDS occurred in 195 EARLI patients (41\%) and 99 VALID patients (29\%). ARDS was independently associated with risk of hospital death in multivariate analysis, even after controlling for severity of illness, as measured by APACHE II (odds ratio [OR] 1.65 (95\% confidence interval [Cl] 1.02, 2.67), $p=0.04$ in EARLl; OR 2.12 (Cl 1.16, 3.92), $p=0.02$ in VALID). Patients with severe ARDS (P/F $<100)$ primarily drove this relationship. The attributable mortality of ARDS was 27\% (CI 14\%, 37\%) in EARLI and 37\% (CI 10\%, 51\%) in VALID. ARDS was independently associated with ICU mortality, hospital length of stay (LOS), ICU LOS, and ventilator-free days.

Conclusions: Development of ARDS among ICU patients with sepsis confers increased risk of ICU and in-hospital mortality in addition to other important outcomes. Clinical trials targeting patients with severe ARDS will be best poised to detect measurable differences in these outcomes.

Keywords: Acute respiratory distress syndrome, Acute lung injury, Sepsis, Mortality

\footnotetext{
*Correspondence: Catherine.auriemma@pennmedicine.upenn.edu

${ }^{1}$ Division of Pulmonary, Allergy, and Critical Care Medicine, Department

of Medicine, Hospital of the University of Pennsylvania, 839 W. Gates, 3600 Spruce Street, Philadelphia, PA 19103-4283, USA

Full author information is available at the end of the article
}

\section{重 Springer}




\section{Introduction}

Acute respiratory distress syndrome (ARDS) is a clinical syndrome in critically ill patients involving acute respiratory failure, hypoxemia, and non-cardiogenic pulmonary edema $[1,2]$. To date, there are no effective pharmacotherapies for ARDS. The attributable mortality for ARDS among patients with sepsis, the most common ARDS risk factor, has not been previously estimated. Empiric estimation of the effect of ARDS on mortality is crucial in this population for the design of future clinical trials.

Proximal causes of death are notoriously difficult to identify in critically ill patients, particularly since most ICU patients die after withdrawal of life-sustaining measures [3, 4]. Given that the supportive therapies shown to reduce mortality in ARDS include primarily a ventilator strategy and prone positioning, it may seem implicit that there exists some modifiable mortality attributable to ARDS related to oxygenation $[5,6]$. However, for decades, it has been clear that refractory hypoxemia accounts for only a small fraction of deaths in ARDS [7, 8]. A recent study showed the majority of deaths of ICU patients with ARDS were not directly related to lung damage [9]. Thus, it has been challenging to determine what proportion of mortality is attributable to ARDS itself (and therefore a potential target for ARDS-focused clinical trials), and what proportion is driven by the underlying ARDS risk factor, comorbidities, or a combination of the two. Quantifying the ARDS-attributable mortality-the excess mortality among patients with ARDS that can be attributed to ARDS - would help inform design of future ARDS clinical trials.

Previous studies examining the impact of ARDS on mortality have shown conflicting results [10-17]. In a large retrospective study of ventilated ICU patients, development of early or late ARDS was not associated with an increase in mortality at 28 days [17]. However, severe ARDS was associated with increased mortality at 2 years. These studies have varied tremendously with regard to patient population and controlling for baseline severity of illness. Notably, many studies focused on trauma populations, a subgroup which may not be representative of patients with other ARDS risk factors [18]. The relevance of older studies to current practice is also unclear, given most were performed before the era of low tidal volume ventilation and modern resuscitation practices. Recent studies have not focused on sepsis, the most common ARDS risk factor.

Using two prospective ICU cohorts, we assessed whether development of ARDS in the current era is independently associated with mortality among medical and surgical ICU patients with sepsis. Limited results from this study were reported in abstract [19].

\section{Take-home message}

In two prospective cohorts of critically ill patients with sepsis, development of ARDS conferred increased risk for hospital mortality, independent of overall severity of illness. This association was driven almost entirely by those patients with severe ARDS. Development of ARDS was also associated with increased intensive care (ICU) mortality, hospital length-of-stay, and ICU length-of-stay.

\section{Methods \\ Participants}

We studied patients from two prospectively enrolled critically ill adult cohorts: (1) Early Assessment of Renal and Lung Injury (EARLI) study and (2) Validating Acute Lung Injury markers for Diagnosis (VALID) study [20-23]. The EARLI cohort includes adult patients admitted from the emergency department to an ICU at either an academic medical center or county hospital in San Francisco, California [20, 22]. The VALID cohort includes adult patients from an academic medical center in Nashville, Tennessee [21, 23]. EARLI was approved by the University of California, San Francisco Institutional Review Board (IRB). VALID was approved by the Vanderbilt IRB. In both cohorts, consent was obtained from patients or their surrogates when possible. Further details about enrollment and consent have been reported and are provided in the online data supplement [20-23].

\section{Primary outcome and additional variables}

We selected patients with sepsis from the EARLI and VALID cohorts. Sepsis was defined as documented or suspected infection in the presence of two or more characteristics of the systemic inflammatory response syndrome [24]. Enrollment and data collection for both cohorts began before the advent of Sepsis 3, and our coding for sepsis reflects the prior definition. Patients were defined as having ARDS if they met Berlin criteria for ARDS on at least 1 day between hospital days one through five in EARLI and between hospital days one through four in VALID [1]. We additionally identified patients who met the American-European Consensus Conference (AECC) criteria for acute lung injury (ALI) during the same time frame [25]. Shock was defined as use of vasopressors within the first $48 \mathrm{~h}$ of ICU stay. Code status at admission was assessed in EARLI based on documented preferences in medical records.

Severity of illness was assessed using APACHE II and SAPS II in both cohorts. In EARLI, APACHE III was also assessed. Modified APACHE scores that exclude points related to oxygenation were generated [10]. The primary outcome in both cohorts was in-hospital mortality. Secondary outcomes included ICU mortality, hospital length of stay (LOS), ICU LOS, and ventilator-free days (VFDs). 
$P / F$ ratios were used to stratify patients by severity of ARDS. Additional detail is provided in the online data supplement.

\section{Statistical methods}

Student's $t$ tests, Pearson's Chi-square test, and MannWhitney-Wilcoxon tests were used to compare baseline variables between cohorts as well as within cohorts stratified by development of ARDS and mortality. We generated a directed acyclic graph (DAG) to illustrate the relationship between ARDS and death and to categorize variables as potential confounders or effect mediators (Supplementary Figure S1) [26]. Multivariate logistic, linear, and zero-inflated negative binomial regression models were developed for primary and secondary outcomes as described in detail in the online data supplement.

Sensitivity analyses were performed in EARLI by restricting analyses to patients with pulmonary sepsis and shock and patients without limitations in code status, and by excluding patients who died within the first 5 days of hospitalization and therefore may have died before development of ARDS was possible. We also performed a sensitivity analysis of hospital and ICU LOS in which we included all participants (not just survivors). In both cohorts, patients, who met the AECC criteria for ALI but were not mechanically ventilated, were excluded from the primary analysis [25]. Sensitivity analyses were also performed by including these patients as cases.

In both cohorts, we determined the attributable fraction of mortality from ARDS $\left(\mathrm{AF}_{\mathrm{ARDS}}\right)$ and the population attributable fraction of mortality from ARDS (population $\mathrm{AF}_{\mathrm{ARDS}}$ ) using methods outlined previously (additional detail in the online supplement) [27-29]. The AF $_{\text {ARDS }}$ is the proportion of deaths attributable to ARDS among all deaths in patients who developed ARDS. The population $\mathrm{AF}_{\mathrm{ARDS}}$ is the proportion of deaths attributable to ARDS among all deaths in the population of patients with sepsis. Estimates were based on indirect standardization, which computes the weighted average of stratum-specific estimates in the reference population, using weights from the study population. Strata were defined by modified APACHE II quartiles. An additional sensitivity analysis was performed among pooled data from EARLI and VALID to assess for a data-driven $P / F$ cutoff for severe ARDS that best captures mortality. A two-sided P value less than 0.05 was considered statistically significant. Analyses were performed using STATA 15 software (College Station, TX) and Proc STDRATE in SAS (v9.4).

\section{Results}

\section{Baseline characteristics and clinical outcomes}

Patient selection is presented in Fig. 1. Table 1 presents baseline characteristics from each cohort. The EARLI population was generally older and more racially diverse compared to the VALID population. Most patients in both cohorts were cared for by a medical ICU service (compared to surgical service). While patients in each cohort had similar modified APACHE II scores, the EARLI cohort had higher SAPS II scores. While the proportion of patients requiring mechanical ventilation was similar, significantly more patients developed ARDS in EARLI compared to VALID. EARLI patients were more likely to meet severe ARDS criteria compared to VALID. Hospital LOS was longer in VALID. ICU and in-hospital mortality were higher in EARLI.

Table 1 also shows each cohort stratified by development of ARDS. In both cohorts, patients who developed ARDS were more likely to have sepsis from a pulmonary source, had higher baseline severity of illness scores, and were more likely to require vasopressor use in the first $48 \mathrm{~h}$ than patients who did not develop ARDS. Patients in both cohorts with ARDS had greater hospital and ICU mortality, longer LOS, and fewer VFDs. Limited data on mechanical ventilation of ARDS patients are presented in Supplemental Table 2.

\section{Comparison of clinical outcomes adjusted for severity of illness \\ EARLI}

Of 474 patients, 137 (29\%) died prior to hospital discharge (Table 2A). Patients who died were older and more likely to have a limit on code status at the time of admission when compared to patients who survived. Patients who died were also more severely ill, with higher modified APACHE II and III scores, higher SAPS II scores, increased vasopressor use in the first $48 \mathrm{~h}$, and more likely to develop ARDS than survivors. Among those who died, 61\% developed ARDS during hospitalization compared with $33 \%$ among survivors $(p<0.001)$.

The unadjusted in-hospital mortality rate was $43 \%$ for patients with ARDS compared to $19 \%$ without (Table 3; $\mathrm{OR}=3.09 ; 95 \%$ CI $2.05,4.66 ; p<0.001)$. In creating adjusted models, no variables in addition to those prespecified (modified APACHE score, age, limitation on code status at admission, and being admitted from a nursing home) met criteria for inclusion in the model as outlined in the methods. In logistic regression adjusted for modified APACHE II, age, limitation on code status, and admission from nursing home, the OR for hospital death among patients with ARDS in EARLI was attenuated by adjustment, but remained statistically significant. In addition, ARDS was associated with increased risk of ICU death in all unadjusted and adjusted models (Table 3A). Results were similarly unchanged when analyses were expanded to the AECC ALI criteria that did not require mechanical ventilation (Supplementary Table S3). 


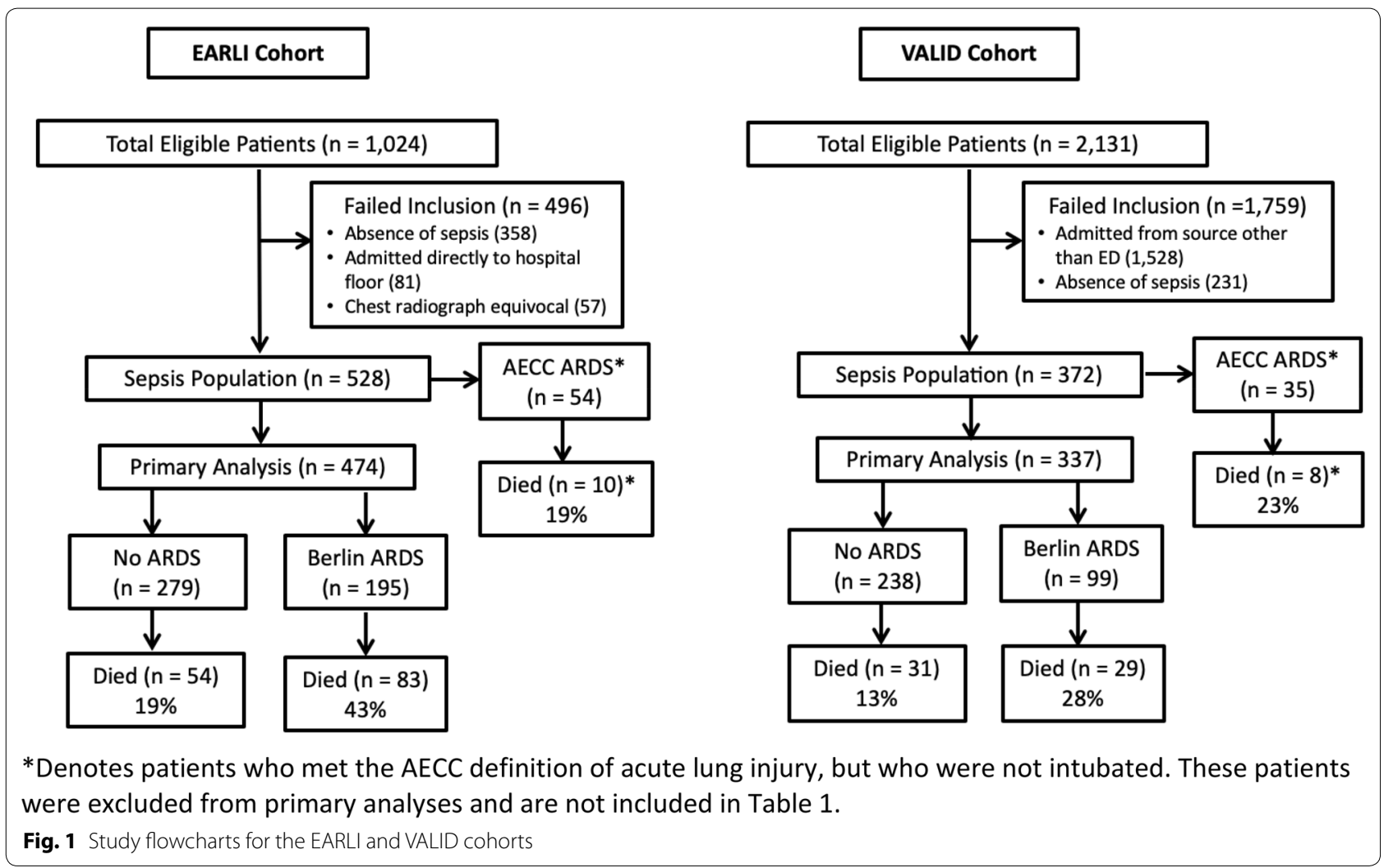

The other sensitivity analyses (excluding patients admitted from a nursing home or having a limitation on code status at admission and excluding patients who died within the first 5 days of hospitalization) did not significantly alter the results (data not shown). Relative to patients with sepsis who did not develop ARDS, patients who developed ARDS had a longer hospital and ICU LOS in both adjusted and unadjusted models, whether limited to only survivors or among all patients (online data supplement Table S4 and S5).

In EARLI, we additionally adjusted for modified APACHE III or unmodified SAPS II in place of modified APACHE II. The association between ARDS and in-hospital mortality was no longer statistically significant when including a modified APACHE III or SAPS II, though the ORs remained similar. For ICU mortality, hospital LOS, and ICU LOS, results were largely unchanged when adjusting for the alternative severity of illness measures (Table 3, Supplementary Tables S3 and S4).

We also performed analyses stratifying by severity of ARDS using the Berlin criteria (1). Severe ARDS conferred independent risk of both hospital and ICU mortality in both unadjusted and adjusted models (Fig. 2, Supplementary Table S6). In adjusted models, severe ARDS was associated with hospital and ICU mortality with ORs of 2.42 (95\% CI 1.24, 4.72; $p=0.01)$ and 3.08
(95\% CI 1.53, 6.21; $p=0.002$ ), respectively. In contrast, mild and moderate ARDS were not independently associated with mortality. In EARLI, the $\mathrm{AF}_{\text {ARDS }}$ was $27 \%(\mathrm{CI}$, $14-37 \%)$ and the population $\mathrm{AF}_{\mathrm{ARDS}}$ was $16 \%(95 \% \mathrm{CI}$, $5-27 \%)$.

\section{VALID}

Of 337 patients, 60 (18\%) died prior to hospital discharge (Table 2B). Patients who died were more likely to be white. They also had higher modified APACHE II scores, SAPS II scores, and increased vasopressor use and were more likely to develop ARDS than survivors. Among those who died, $48 \%$ developed ARDS during hospitalization compared with $25 \%$ among survivors $(p<0.001)$.

The unadjusted in-hospital mortality rate was $28 \%$ for patients with ARDS compared to $13 \%$ for patients without $(\mathrm{OR}=2.77$; 95\% CI 1.56, 4.91; $p=0.001)$ (Table 3B). Similar to findings in EARLI, in multivariate logistic regression, development of ARDS in the overall VALID sample was an independent risk factor for both hospital and ICU death. Results were similar in models using SAPS II as a marker for severity of illness. Again, results were largely unchanged when analyses were expanded to include the AECC ALI criteria that did not require mechanical ventilation (Supplementary Table S3) or 
Table 1 Baseline characteristics of EARLI and VALID cohorts, together and stratified by ARDS

\begin{tabular}{|c|c|c|c|c|c|c|c|c|}
\hline \multirow[t]{2}{*}{ Clinical variable* } & \multicolumn{2}{|l|}{ All patients } & \multicolumn{3}{|c|}{ EARLI $(n=474)$} & \multicolumn{3}{|l|}{ VALID $(n=337)$} \\
\hline & EARLI $(n=474)$ & VALID $(n=337)$ & $\begin{array}{l}\text { No ARDS } \\
(n=279)\end{array}$ & $\begin{array}{l}\text { ARDS } \\
(n=195)\end{array}$ & $p$ value & No ARDS $(n=238)$ & ARDS $(n=99)$ & $p$ value \\
\hline Age, years & $66 \pm 17$ & $56 \pm 16$ & $65 \pm 17$ & $68 \pm 16$ & 0.07 & $56 \pm 15$ & $53 \pm 17$ & 0.11 \\
\hline Male gender, $\%$ & $255(54)$ & $169(50)$ & $153(55)$ & $102(52)$ & 0.59 & $117(49)$ & $52(53)$ & 0.57 \\
\hline White race, \% & $239(50)$ & $267(79)$ & $139(50)$ & $100(51)$ & 0.75 & $182(76)$ & $85(86)$ & 0.05 \\
\hline $\begin{array}{l}\text { Any limitation on code status at } \\
\text { admission, \% }\end{array}$ & $88(19)$ & - & $52(19)$ & $36(18)$ & 0.96 & - & - & - \\
\hline Admitted from nursing facility, $\%$ & $96(20)$ & - & $54(19)$ & $42(22)$ & 0.56 & - & - & - \\
\hline Pulmonary sepsis, $\%$ & $277(58)$ & $172(51)$ & $125(45)$ & $152(78)$ & $<0.001$ & $98(41)$ & $74(75)$ & $<0.001$ \\
\hline Primary Service Medicine, \% & $429(91)$ & $319(95)$ & $246(88)$ & $183(94)$ & 0.07 & $228(96)$ & $91(92)$ & 0.15 \\
\hline APACHE II score & $27 \pm 9$ & $27 \pm 9$ & $24 \pm 8$ & $31 \pm 10$ & $<0.001$ & $25 \pm 9$ & $30 \pm 8$ & $<0.001$ \\
\hline Modified APACHE II score ${ }^{\dagger}$ & $25 \pm 9$ & $25 \pm 8$ & $23 \pm 7$ & $29 \pm 9$ & $<0.001$ & $24 \pm 8$ & $28 \pm 8$ & $<0.001$ \\
\hline APACHE III score & $95 \pm 39$ & - & $83 \pm 33$ & $113 \pm 40$ & $<0.001$ & - & - & - \\
\hline Modified APACHE III score ${ }^{\dagger}$ & $90 \pm 36$ & - & $80 \pm 31$ & $104 \pm 38$ & $<0.001$ & - & - & - \\
\hline SAPS $\|$ & $53 \pm 22$ & $50 \pm 18$ & $46 \pm 18$ & $62 \pm 23$ & $<0.001$ & $46 \pm 17$ & $58 \pm 19$ & $<0.001$ \\
\hline Vasopressor use in first 48 h, \% & $276(58)$ & $175(52)$ & $139(50)$ & $137(70)$ & $<0.001$ & $112(47)$ & $63(64)$ & 0.01 \\
\hline Mechanical ventilation, $\%$ & $275(58)$ & $205(61)$ & $107(38)$ & $168(100)$ & $<0.001$ & $106(43)$ & $99(100)$ & $<0.001$ \\
\hline ARDS, \% & $195(41)$ & $99(29)$ & - & - & - & - & - & - \\
\hline $\begin{array}{l}\text { Mild ARDS, defined as PF or } \\
\text { SF }>200-300, \%\end{array}$ & $43(22)$ & $32(32)$ & - & - & - & - & - & - \\
\hline $\begin{array}{l}\text { Moderate ARDS, defined as PF } \\
\text { or SF } 100-200, \%\end{array}$ & $76(39)$ & $42(42)$ & - & - & - & - & - & - \\
\hline $\begin{array}{l}\text { Severe ARDS, defined as PF or } \\
\text { SF }<100, \%\end{array}$ & $76(39)$ & $25(25)$ & - & - & - & - & - & - \\
\hline Hospital LOS & $8(5,13)$ & $9(6,15)$ & $7(5,11)$ & $9(5,16)$ & 0.004 & $9(6,13)$ & $12(7,20)$ & 0.001 \\
\hline Hospital LOS & $8(5,13)$ & $10(7,17)$ & $7(5,11)$ & $10(7,19)$ & $<0.001$ & $9(6,14)$ & $15(10,23)$ & $<0.001$ \\
\hline ICU LOS & $4(3,7)$ & $5(3,9)$ & $4(3,5)$ & $5(3,11)$ & $<0.001$ & $4(3,6)$ & $8(5,13)$ & $<0.001$ \\
\hline ICU LOS & $4(3,6)$ & $4(3,8)$ & $4(3,5)$ & $5(4,11)$ & $<0.001$ & $4(3,5)$ & $9(5,13)$ & $<0.001$ \\
\hline Ventilator-free days & $25(0,28)$ & $25(16,28)$ & $28(23,28)$ & $16(0,26)$ & $<0.001$ & $28(24,28)$ & $20(1,24)$ & $<0.001$ \\
\hline ICU mortality, \% & $105(22)$ & $46(14)$ & $36(13)$ & $69(35)$ & $<0.001$ & $21(9)$ & $25(25)$ & $<0.001$ \\
\hline Hospital mortality, \% & $137(29)$ & $60(18)$ & $54(19)$ & $83(43)$ & $<0.001$ & $31(13)$ & $29(28)$ & $<0.001$ \\
\hline
\end{tabular}

Table excludes all patients who met the AECC definition of acute lung injury but were not intubated

LOS length of stay

*Data shown as mean \pm standard deviation, number (percent), or median (interquartile range) as appropriate

+ Modified APACHE scores exclude points related to oxygenation

* Restricted to survivors

excluding patients who died within the first 5 days (data not shown).

As in EARLI, development of ARDS conferred increased risk of prolonged hospital and ICU LOS as well as fewer VFDs (Supplementary Tables S3 and S4). In addition, the association between ARDS and mortality was also stratified by severity of ARDS using the Berlin criteria (1). In VALID, severe ARDS again conferred independent risk of in-hospital mortality in unadjusted and adjusted models (Fig. 2, Supplementary Table S6). The OR for hospital and ICU mortality adjusted for APACHE II was 2.12 (95\% CI 1.16, 3.92; $p=0.02)$ and 2.67 (95\% CI 1.35, 5.27; $p=0.01)$, respectively. The analysis of in-hospital mortality did not reach statistical significance in the model adjusted for SAPS II, though ORs were similar to other models and those in EARLI (online data Supplementary Table S5). In VALID, the $\mathrm{AF}_{\mathrm{ARDS}}$ was $37 \%(10-51 \%)$ and the population $\mathrm{AF}_{\mathrm{ARDS}}$ was $18 \%$ (95\% CI, 0.3\%, 32\%).

In a sensitivity analysis performed on pooled data from EARLI and VALID to determine a data-driven threshold for capturing mortality of severe ARDS, the risk of mortality appeared to plateau at a $P / F$ ratio of 120 (Supplementary Figure S3). 
Table 2 Patient characteristics stratified by in-hospital mortality, EARLI and VALID cohorts

\begin{tabular}{|c|c|c|c|}
\hline Clinical variable* & Survived ( $n=337$ ) & Died $(n=137)$ & $p$ value \\
\hline \multicolumn{4}{|l|}{ EARLI patient characteristics } \\
\hline Age, years & $64 \pm 17$ & $70 \pm 15$ & $<0.001$ \\
\hline Male gender, \% & $176(53)$ & $79(57)$ & 0.28 \\
\hline White race, $\%$ & $174(52)$ & $65(47)$ & 0.41 \\
\hline Any limitation on code status at admission, \% & $55(16)$ & $33(24)$ & 0.05 \\
\hline Admitted from nursing facility, $\%$ & $65(19)$ & $31(23)$ & 0.41 \\
\hline Pulmonary sepsis, \% & $188(56)$ & $89(65)$ & 0.07 \\
\hline APACHE II score & $24 \pm 8$ & $34 \pm 9$ & $<0.001$ \\
\hline Modified APACHE II score ${ }^{\dagger}$ & $22 \pm 7$ & $32 \pm 8$ & $<0.001$ \\
\hline APACHE III score & $82 \pm 30$ & $129 \pm 39$ & $<0.001$ \\
\hline Modified APACHE III score ${ }^{\dagger}$ & $78 \pm 28$ & $121 \pm 37$ & $<0.001$ \\
\hline SAPS $\|$ & $45 \pm 17$ & $71 \pm 20$ & $<0.001$ \\
\hline Vasopressor use in first 48 h, \% & $172(51)$ & $104(76)$ & $<0.001$ \\
\hline Mechanically ventilated, \% & $164(49)$ & $111(81)$ & $<0.001$ \\
\hline ARDS, $\%$ & $112(33)$ & $83(61)$ & $<0.001$ \\
\hline Hospital LOS & $8(5,13)$ & $7(3,13)$ & 0.002 \\
\hline ICU LOS & $4(3,6)$ & $5(3,10)$ & 0.11 \\
\hline Clinical variable* & Survived $(n=277)$ & Died $(n=60)$ & $p$ value \\
\hline \multicolumn{4}{|l|}{ VALID patient characteristics } \\
\hline Age, years & $55 \pm 16$ & $58 \pm 16$ & 0.23 \\
\hline Male gender, \% & $138(50)$ & $31(52)$ & 0.80 \\
\hline White race, $\%$ & $213(77)$ & $54(90)$ & 0.02 \\
\hline Pulmonary sepsis, \% & $134(48)$ & $38(63)$ & 0.04 \\
\hline APACHE II score & $26 \pm 8$ & $32 \pm 8$ & $<0.001$ \\
\hline Modified APACHE II score ${ }^{\dagger}$ & $24 \pm 8$ & $30 \pm 8$ & $<0.001$ \\
\hline SAPS $\|$ & $47 \pm 17$ & $62 \pm 19$ & $<0.001$ \\
\hline Vasopressor use in first 48 h, \% & $133(48)$ & $42(70)$ & 0.002 \\
\hline Mechanically ventilated, \% & $152(55)$ & $53(88)$ & $<0.001$ \\
\hline ARDS, $\%$ & $70(25)$ & $29(48)$ & 0.001 \\
\hline Hospital LOS & $10(7,17)$ & $6.5(4,10)$ & $<0.001$ \\
\hline ICU LOS & $4(3,8)$ & $6(4,9.5)$ & 0.01 \\
\hline
\end{tabular}

*Data shown as mean \pm standard deviation, number (percent), or median (interquartile range) as appropriate

+ Modified APACHE scores exclude points related to oxygenation

\section{Discussion}

We determined the association between development of ARDS and mortality in two separate ICU cohorts of critically ill patients with sepsis. In both cohorts, development of ARDS was independently associated with higher hospital and ICU mortality, accrual of fewer VFDs, and prolonged hospital and ICU LOS. While it may not be surprising that severe ARDS portends a worse prognosis than moderate or mild disease, the varied ability to detect statistically significant mortality differences based on severity of ARDS is, to our knowledge, a novel finding. Most recently, Fuchs et al. showed no detectable difference in 28-day mortality among ventilated patients with or without ARDS, but did find that severe ARDS served as a risk factor for 2-year mortality [17]. Our significant results for in-hospital mortality compared to the results of Fuchs et al. may relate to differences in overall severity of illness and our focus on a predominantly medical ICU population with sepsis.

Our findings are directly relevant to the growing interest in prognostic enrichment for improving critical care trial design. Prognostic enrichment is defined as preferentially targeting enrollment of patients with the highest rates of disease-attributable (and, hopefully, modifiable) outcomes-in this case, mortality. With increasing recognition that current definitions of sepsis and ARDS do not identify patients with uniform 
Table 3 Association of ARDS with mortality in unadjusted and adjusted models, EARLI and VALID cohorts

\begin{tabular}{|c|c|c|}
\hline EARLI logistic regression models $(n=474)$ & OR $(95 \% \mathrm{Cl})$ & $p$ value \\
\hline Unadjusted model of ARDS for in-hospital mortality & $3.09(2.05,4.66)$ & $<0.001$ \\
\hline Adjusted for modified APACHE II* & $1.65(1.02,2.67)$ & 0.04 \\
\hline Adjusted for modified APACHE II** & $1.61(0.98,2.64)$ & 0.06 \\
\hline Adjusted for SAPS $\|^{*}$ & $1.48(0.9,2.44)$ & 0.12 \\
\hline Unadjusted model of ARDS for ICU mortality & $3.70(2.34,5.84)$ & $<0.001$ \\
\hline Adjusted for modified APACHE II* & $2.05(1.23,3.44)$ & 0.01 \\
\hline Adjusted for modified APACHE III* & $2.03(1.2,3.45)$ & 0.01 \\
\hline Adjusted for SAPS $\|^{*}$ & $1.85(1.1,3.13)$ & 0.02 \\
\hline VALID logistic regression models $(n=337)$ & OR $(95 \% \mathrm{Cl})$ & $p$ \\
\hline Unadjusted model of ARDS for in-hospital mortality & $2.77(1.56,4.91)$ & 0.001 \\
\hline Adjusted for modified APACHE $\|^{+}$ & $2.12(1.16,3.92)$ & 0.02 \\
\hline Adjusted for SAPS $\|^{\dagger}$ & $1.79(0.95,3.36)$ & 0.07 \\
\hline Unadjusted model of ARDS for ICU mortality & $3.49(1.85,6.6)$ & $<0.001$ \\
\hline Adjusted for modified APACHE II ${ }^{\dagger}$ & $2.67(1.35,5.27)$ & 0.01 \\
\hline Adjusted for SAPS $\|^{+}$ & $2.14(1.06,4.33)$ & 0.03 \\
\hline
\end{tabular}

Modified APACHE scores exclude points related to oxygenation

*In addition to severity of illness variable listed in the table, adjusted models include age, limitation on code status at admission, and being admitted from a nursing home

${ }^{\dagger}$ In addition to severity of illness variable listed in the table, adjusted models include age

and distinct disease processes, and that this heterogeneity may be confounding clinical trials, prognostic enrichment may offer one reasonable approach [30]. While targeting patients with more severe ARDS has had some success $[6,31]$, this study provides the first empiric evidence to support such a strategy. Our findings suggest that prognostic enrichment focusing on patients with severe ARDS, either as defined by the Berlin definition of $P / F<100$, or perhaps by $P / F<120$, as suggested by our data-driven comparison of mortality risk, may identify individuals most likely to die from factors related specifically to ARDS, and therefore may provide a population in which we are better able to identify beneficial treatments. This finding is consistent with a prior autopsy study which reported that patients with severe ARDS were more likely to experience refractory hypoxemia prior to death and have diffuse alveolar damage than patients with mild or moderate disease who were more likely to die of refractory shock [32].

We also estimated $\mathrm{AF}_{\mathrm{ARDS}}$ and population $\mathrm{AF}_{\mathrm{ARDS}}$ and found similar results in both cohorts. Attributable fraction of mortality is the proportion of deaths that can be statistically attributed to an underlying cause, in this case, ARDS [28]. Population attributable fraction of mortality is the proportion of deaths within a cohort of patients, all of whom are at risk of the underlying cause in question: In this case, the proportion of deaths due to ARDS among all patients with sepsis. The $\mathrm{AF}_{\mathrm{ARDS}}$ was
$27 \%$ in EARLI and $37 \%$ in VALID. The population $\mathrm{AF}_{\mathrm{ARDS}}$ was $16 \%$ in EARLI and 18\% in VALID. It is helpful to put these results in the context of other studies estimating attributable mortality of important critical care diseases. The attributable fraction of mortality from sepsis was recently estimated at $15 \%$ [33]. The population attributable fraction of mortality from ICU-acquired infections and ICU delirium has been estimated at $11 \%$ and $7 \%$, respectively [29, 34]. While ARDS may confer mortality fractions notably higher than these other important ICU conditions, any absolute changes in mortality by fully treating or preventing ARDS would still be relatively low. As recently illustrated by Shankar-Hari et al., these data have major implications for considering the size of clinical trials necessary to detect such changes [33].

This study has several strengths. First, it includes two large and diverse prospective cohorts of critically ill patients from distinct centers. The similarity of the association between ARDS and in-hospital mortality in these very different populations strengthens the validity of our findings and suggests generalizability. Second, we found relatively consistent results within each cohort when adjusting for up to three distinct severity of illness metrics. Third, in developing adjusted models, we searched methodically for possible confounders and did not find other contributory variables. Fourth, all patients were meticulously phenotyped for both sepsis and ARDS. Finally, in contrast to most studies assessing ARDS and attributable mortality, this study expands the populations 


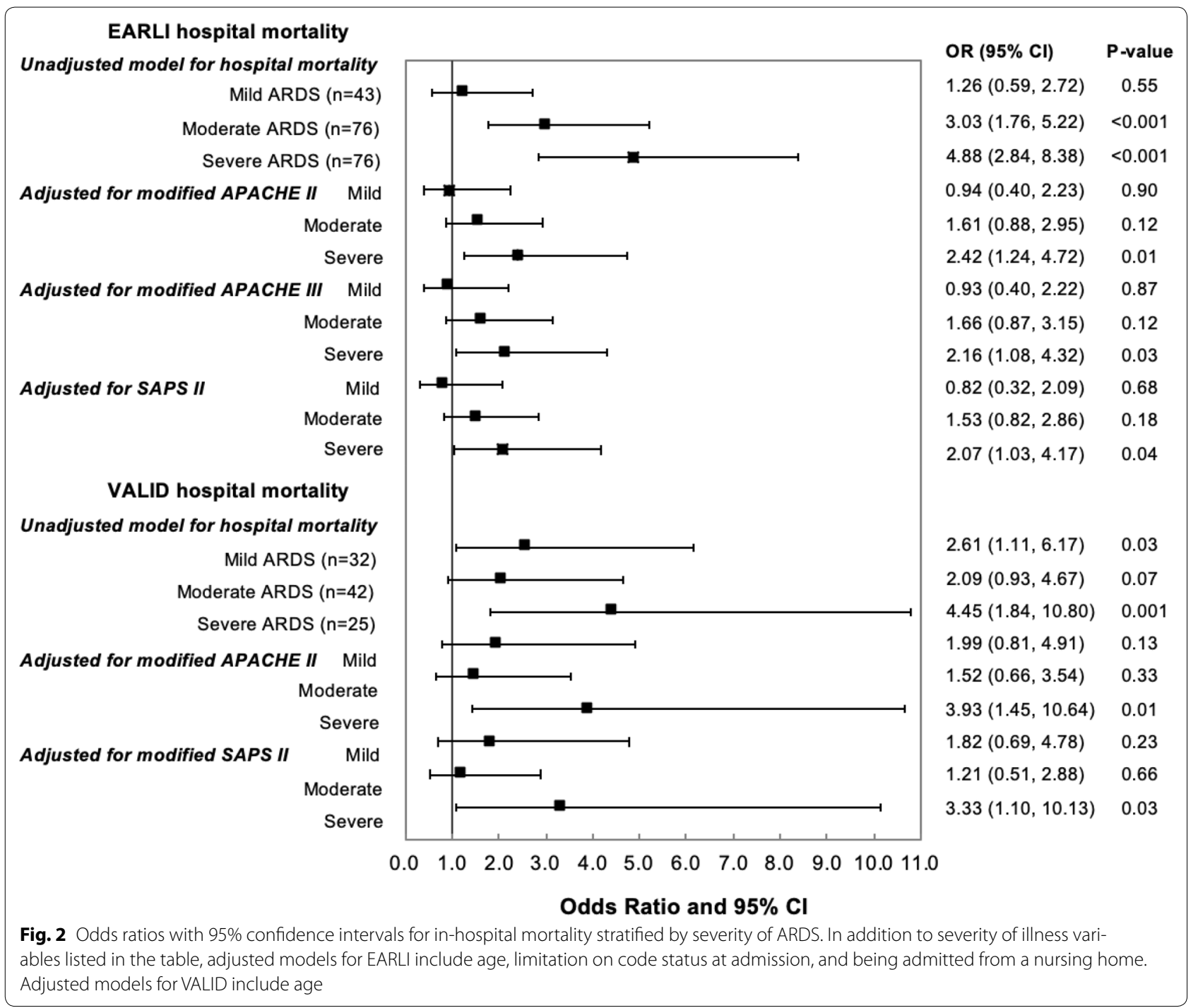

to include both medical and surgical patients, thereby increasing generalizability of results beyond previously published studies.

This study has limitations. First, there were some discrepancies in specific data collected in each cohort. Most notably, we did not have information on code status or admission from a nursing home in the VALID population. However, incorporation of these variables did not significantly affect any of the EARLI models. In addition, we were unable to generate APACHE III scores from the VALID data. To better align the cohorts, we chose to utilize APACHE II for our primary analyses. While we adjusted for multiple confounders, the possibility of residual confounding remains. Specifically, we did not control for measures of other acute organ failures beyond what is captured in APACHE and SAPS scores. As depicted in our DAG, we hypothesize that other acute organ failures may lie on the indirect causal path between ARDS and death, and so were not included in our models. However, if the association between ARDS and mortality is driven predominantly by other organ failures (such as renal or hepatic) that develop later in a patient's ICU stay, or those variables are instead predominantly confounders, our analysis may not precisely capture that relationship. This caveat is important because some prior studies have identified increased mortality in ARDS with other organ failures or comorbidities $[35,36]$. Our study does not explain the cause of the observed higher mortality in patients with severe ARDS, but future studies focusing on severe ARDS should explore this question. Because we began enrollment and data collection for both cohorts before the advent of Sepsis 3, our coding for sepsis reflects the prior definition. However, as we enrolled critically ill 
patients, it is unlikely that our patients would not fulfill the more recent criteria for sepsis [37]. Finally, as our study focused on patients with sepsis, the most common ARDS risk factor, findings may not be generalizable to patients with other risk factors for ARDS.

This study provides important new evidence about ARDS-attributable mortality in patients with sepsis and quantifies the $\mathrm{AF}_{\mathrm{ARDS}}$. In two separate cohorts of ICU patients with sepsis, ARDS is independently associated with hospital and ICU mortality as well as VFDs, ICU LOS, and hospital LOS. Importantly, patients with the most severe ARDS primarily drove the relationship between ARDS and mortality. These findings suggest that to successfully identify novel therapeutics or changes in practice that may impact mortality, clinical trials for ARDS will require significantly larger study populations or should focus primarily on patients with severe ARDS.

\section{Electronic supplementary material}

The online version of this article (https://doi.org/10.1007/s00134-020-06010-9) contains supplementary material, which is available to authorized users.

\begin{abstract}
Author details
${ }^{1}$ Division of Pulmonary, Allergy, and Critical Care Medicine, Department of Medicine, Hospital of the University of Pennsylvania, 839 W. Gates, 3600 Spruce Street, Philadelphia, PA 19103-4283, USA. ${ }^{2}$ Palliative and Advanced IIIness Research (PAIR) Center, University of Pennsylvania, Philadelphia, PA, USA. ${ }^{3}$ Cardiovascular Research Institute, University of California, San Francisco, CA, USA. ${ }^{4}$ Department of Psychiatry, University of California, San Francisco, USA. ${ }^{5}$ Department of Biochemistry and Biophysics, University of California, San Francisco, USA. ${ }^{6}$ Virginia Tech Carilion School of Medicine, Roanoke, VA, USA. ${ }^{7}$ Department of Medicine, University of California, San Francisco, USA. ${ }^{8}$ Department of Pulmonary and Critical Care Medicine, Zuckerberg San Francisco General Hospital and Trauma Center, University of California, San Francisco, USA. ${ }^{9}$ University of Colorado School of Medicine, Aurora, CO, USA. ${ }^{10}$ Division of Pulmonary and Critical Care Medicine, Department of Medicine, University of California, San Francisco, USA. ${ }^{11}$ Division of Nephrology, Department of Medicine, University of California, San Francisco, USA. ${ }^{12}$ Department of Anesthesia, University of California San Francisco, San Francisco, CA, USA.

${ }^{13}$ Departments of Medicine and Pathology, Microbiology, and Immunology, Vanderbilt University, Nashville, TN, USA.
\end{abstract}

\section{Acknowledgements}

We wish to thank the patients and their families who participated in this study. We also thank the ICU staff at both of our cohort sites, and in particular the ICU nurses. Dr. Calfee received financial support for this study from the National Heart Lung, and Blood Institute (HL140026). Dr. Ware received financial support for this study from the National Heart Lung, and Blood Institute (HL103836 and HL135849).

\section{Compliance with ethical standards}

\section{Conflicts of interest}

On behalf of all authors, the corresponding author states that there is no conflict of interest.

\section{Publisher's Note}

Springer Nature remains neutral with regard to jurisdictional claims in published maps and institutional affiliations.

Received: 7 August 2019 Accepted: 11 March 2020 Published online: 23 March 2020

\section{References}

1. Ranieri VM, Rubenfeld GD, Thompson BT et al (2012) Acute respiratory distress syndrome: the Berlin definition. JAMA, J Am Med Assoc 307:2526-2533. https://doi.org/10.1001/jama.2012.5669

2. Matthay MA, Zemans RL, Zimmerman GA et al (2019) Acute respiratory distress syndrome. Nat Rev 5:1-22. https://doi.org/10.1016/B978-0-32344887-1.00038-9

3. Smedira NG, Evans BH, Grais LS et al (1990) Withholding and withdrawal of life support from the critically III. N Engl J Med 322:309-315. https:// doi.org/10.1056/NEJM199002013220506

4. Prendergast TJ, Luce JM (1997) Increasing incidence of withholding and withdrawal of life support from the critically ill. Am J Respir Crit Care Med 155:15-20. https://doi.org/10.1164/ajrccm.155.1.9001282

5. The Acute Respiratory Distress Syndrome Network, Brower RG, Matthay MA et al (2000) Ventilation with lower tidal volumes as compared with traditional tidal volumes for acute lung injury and the acute respiratory distress syndrome. N Engl J Med 342:1301-1308. https://doi.org/10.1056/ NEJM200005043421801

6. Guérin C, Reignier J, Richard J-C et al (2013) Prone positioning in severe acute respiratory distress syndrome. N Engl J Med 368:2159-2168. https ://doi.org/10.1056/NEJMoa1214103

7. Montgomery AB, Stager MA, Carrico CJ, Hudson LD (1985) Causes of mortality in patients with the adult respiratory distress syndrome. Am Rev Respir Dis 132:485-489. https://doi.org/10.1164/arrd.1985.132.3.485

8. Stapleton RD, Wang BM, Hudson LD et al (2005) Causes and timing of death in patients with ARDS. Chest 128:525-532. https://doi.org/10.1378/ chest.128.2.525

9. Villar J, Martínez D, Mosteiro F et al (2018) Is overall mortality the right composite endpoint in clinical trials of acute respiratory distress syndrome? Crit Care Med 46:892-899. https://doi.org/10.1097/CCM.00000 00000003022

10. Shah CV, Localio AR, Lanken PN et al (2008) The impact of development of acute lung injury on hospital mortality in critically ill trauma patients. Crit Care Med 36:2309-2315. https://doi.org/10.1097/CCM.0b013e3181 $80 \mathrm{dc} 74$

11. Salim A, Martin M, Constantinou C et al (2006) Acute respiratory distress syndrome in the trauma intensive care unit: morbid but not mortal. Arch Surg 141:655-658. https://doi.org/10.1001/archsurg.141.7.655

12. Treggiari MM, Hudson LD, Martin DP et al (2004) Effect of acute lung injury and acute respiratory distress syndrome on outcome in critically ill trauma patients. Crit Care Med 32:327-331. https://doi.org/10.1097/01. CCM.0000108870.09693.42

13. Eberhard LW, Morabito DJ, Matthay MA et al (2000) Initial severity of metabolic acidosis predicts the development of acute lung injury in severely traumatized patients. Crit Care Med 28:125-131. https://doi. org/10.1097/00003246-200001000-00021

14. Eggimann P, Harbarth S, Ricou B et al (2003) Acute respiratory distress syndrome after bacteremic sepsis does not increase mortality. Am J Respir Crit Care Med 167:1210-1214. https://doi.org/10.1164/rccm.20021 0-11960C

15. Davidson TA, Rubenfeld GD, Caldwell ES et al (1999) The effect of acute respiratory distress syndrome on long-term survival. Am J Respir Crit Care Med 160:1838-1842

16. Hudson LD, Milberg JA, Anardi D, Maunder RJ (1995) Clinical risks for development of the acute respiratory distress syndrome. Am J Respir Crit Care Med 151:293-301. https://doi.org/10.1164/ajrccm.151.2.7842182

17. Fuchs $L$, Feng $M$, Novack $V$ et al (2019) The effect of ARDS on survival: do patients die from ARDS or with ARDS? J Intensive Care Med 34:374-382. https://doi.org/10.1177/0885066617717659

18. Calfee CS, Eisner MD, Ware LB et al (2007) Trauma-associated lung injury differs clinically and biologically from acute lung injury due to other clinical disorders. Crit Care Med 25:2243-2250. https://doi.org/10.1097/01. CCM.0000280434.33451.87

19. Auriemma CL, Zhuo H, Deiss T, et al (2016) Acute respiratory distress syndrome does not independently predict mortality among all critically ill patients with sepsis. In: American Thoracic Society. p San Francisco

20. Agrawal A, Matthay MA, Kangelaris KN et al (2013) Plasma angiopoietin-2 predicts the onset of acute lung injury in critically ill patients. Am J Respir Crit Care Med 187:736-742. https://doi.org/10.1164/rccm.201208-14600 C 
21. O'Neal HR, Koyama T, Koehler EAS et al (2011) Prehospital statin and aspirin use and the prevalence of severe sepsis and ALI/ARDS. Crit Care Med 39:1343-1350. https://doi.org/10.1097/CCM.0b013e3182120992.Preho spital

22. Kangelaris KN, Prakash A, Liu KD et al (2015) Increased expression of neutrophil-related genes in patients with early sepsis-induced ARDS. Am J Physiol Cell Mol Physiol 208:L1102-L1113. https://doi.org/10.1152/ajplu ng.00380.2014

23. Russell DW, Janz DR, Emerson WL et al (2017) Early exposure to hyperoxia and mortality in critically ill patients with severe traumatic injuries. BMC Pulm Med 17:29. https://doi.org/10.1186/s12890-017-0370-1

24. Bone RC, Balk RA, Cerra FB et al (1992) Definitions for sepsis and organ failure and guidelines for the use of innovative therapies in sepsis. The ACCP/SCCM Consensus Conference Committee. American College of Chest Physicians/Society of Critical Care Medicine. Chest 101:1644-1655. https://doi.org/10.1378/chest.101.6.1644

25. Bernard GR, Artigas A, Brigham KL et al (1994) The American-European Consensus Conference on ARDS. Definitions, mechanisms, relevant outcomes, and clinical trial coordination. Am J Respir Crit Care Med 149:818-824. https://doi.org/10.1164/ajrccm.149.3.7509706

26. Lederer DJ, Bell SC, Branson RD et al (2019) Control of confounding and reporting of results in causal inference studies. Guidance for authors from editors of respiratory, sleep, and critical care journals. Ann Am Thorac Soc 16:22-28. https://doi.org/10.1513/annalsats.201808-564ps

27. Bruzzi P, Green SB, Byar DP et al (1985) Estimating the population attributable risk for multiple risk factors using case-control data. Am J Epidemiol 122:904-914. https://doi.org/10.1093/oxfordjournals.aje.a114174

28. Wacholder S, Benichou J, Heineman EF et al (1994) Attributable risk: advantages of a broad definition of exposure. Am J Epidemiol 140:303309. https://doi.org/10.1093/oxfordjournals.aje.a117252

29. van Vught LA, Klein Klouwenberg PMC, Spitoni C et al (2016) Incidence, risk factors, and attributable mortality of secondary infections in the intensive care unit after admission for sepsis. JAMA 315:1469-1479. https ://doi.org/10.1001/jama.2016.2691

30. Prescott HC, Calfee CS, Taylor Thompson B et al (2016) Toward smarter lumping and smarter splitting: rethinking strategies for sepsis and acute respiratory distress syndrome clinical trial design. Am J Respir Crit Care Med 194:147-155. https://doi.org/10.1164/rccm.201512-2544CP

31. Papazian L, Forel J-M, Gacouin A et al (2010) Neuromuscular blockers in early acute respiratory distress syndrome. N Engl J Med 363:1107-1116. https://doi.org/10.1056/NEJMoa1005372

32. Thille AW, Esteban A, Fernández-Segoviano P et al (2013) Comparison of the berlin definition for acute respiratory distress syndrome with autopsy. Am J Respir Crit Care Med 187:761-767. https://doi.org/10.1164/ rccm.201211-19810C

33. Shankar-Hari M, Harrison DA, Rowan KM, Rubenfeld GD (2018) Estimating attributable fraction of mortality from sepsis to inform clinical trials. J Crit Care 45:33-39. https://doi.org/10.1016/j.jcrc.2018.01.018

34. Klein Klouwenberg PMC, Zaal IJ, Spitoni C et al (2014) The attributable mortality of delirium in critically ill patients: prospective cohort study. BMJ 349:g6652. https://doi.org/10.1136/bmj.g6652

35. Liu KD, Glidden DV, Eisner MD et al (2007) Predictive and pathogenetic value of plasma biomarkers for acute kidney injury in patients with acute lung injury. Crit Care Med 35:2755-2761

36. Doyle RL, Szaflarski N, Modin GW et al (1995) Identification of patients with acute lung injury: predictors of mortality. Am J Respir Crit Care Med 152:1818-1824. https://doi.org/10.1164/ajrccm.152.6.8520742

37. Singer M, Deutschman CS, Seymour CW et al (2016) The third international consensus definitions for sepsis and septic shock (Sepsis-3) HHS public access. JAMA 315:801-810. https://doi.org/10.1001/ jama.2016.0287 